\title{
Chronic consumption of alcohol increases alveolar bone loss
}

\author{
Juliano Milanezi de Almeida ${ }^{10 *}$, Victor Fabrizio Cabrera Pazmino ${ }^{10}$, Vivian Cristina \\ Noronha Novaes ${ }^{1 \ddagger}{ }^{1 \ddagger}$, Suely Regina Mogami Bomfim ${ }^{2 \ddagger}$, Maria José Hitomi Nagata ${ }^{1 \ddagger}$, Fred \\ Lucas Pinto Oliveira ${ }^{1 \ddagger}$, Henrique Rinald Matheus ${ }^{1 \neq}$, Edilson Ervolino ${ }^{3 \odot}$
}

1 Department of Diagnosis and Surgery-Division of Periodontics, São Paulo State University (Unesp), School of Dentistry, Araçatuba, SP, Brazil, 2 Department of Clinic, Surgery and Animal Reproduction, São Paulo State University (Unesp), School of Veterinary Medicine, Araçatuba, SP, Brazil, 3 Department of Basic Sciences, São Paulo State University (Unesp), School of Dentistry, Araçatuba, SP, Brazil

ه These authors contributed equally to this work. $\ddagger$ These authors also contributed equally to this work.

* jumilanezi@ hotmail.com

\section{Abstract}

\section{OPEn ACCESS}

Citation: de Almeida JM, Pazmino VFC, Novaes VCN, Bomfim SRM, Nagata MJH, Oliveira FLP, et al. (2020) Chronic consumption of alcohol increases alveolar bone loss. PLOS ONE 15(8): e0232731. https://doi.org/10.1371/journal. pone. 0232731

Editor: Hsu-Shan Huang, College of Medical Science and Technology, Taipei Medical University, TAIWAN

\section{Received: April 18, 2020}

Accepted: July 22, 2020

Published: August 20, 2020

Copyright: ๑ 2020 de Almeida et al. This is an open access article distributed under the terms of the Creative Commons Attribution License, which permits unrestricted use, distribution, and reproduction in any medium, provided the original author and source are credited.

Data Availability Statement: All relevant data are within the paper and its Supporting Information files.

Funding: VFC Pazmino thanks the Brazilian Federal Agency for the Support and Evaluation of Graduate Education (Coordenação de Aperfeiçoamento de Pessoal de Nível Superior - CAPES) for the scholarship and JM de Almeida thanks the Fundunesp-PROPE 0096/004/13 for assistance in the development of this research.
This study evaluated the effects of the chronic consumption of different concentrations of alcohol on the experimental periodontitis (EP). 160 rats were divided into 4 groups: (EP-NT) rats with EP and no alcohol exposure; (EP-A14) rats with EP exposed to 14\% alcohol; (EPA25) rats with EP exposed to $25 \%$ alcohol; (EP-A36) rats with EP exposed to $36 \%$ alcohol. The animals from the EP-A14, EP-A25 and EP-A36 groups were subjected to different concentrations of alcohol 30 days before EP induction. The histological characteristics, percentage of bone in the furcation (PBF) and bone metabolism in the furcation region were evaluated. The PBF and tartrate-resistant acid phosphatase (TRAP) data were subjected to statistical analysis. The EP-A14, EP-A25 and EP-A36 groups had lower PBFs compared with the EP-NT group. A more severe inflammatory process and a greater number of TRAP+ cells were also observed. In the EP-A14, EP-A25 and EP-A36 groups, the inflammatory process became more severe as the ingested alcoholic concentration increased. An increase in RANKL immunolabeling and a significantly higher number of TRAP+ cells were also observed. We conclude that chronic alcohol consumption increases the severity of experimental periodontitis in a dose-dependent manner by increasing the magnitude of local inflammatory responses and stimulating alveolar bone resorption.

\section{Introduction}

Periodontitis is a multifactorial infectious-inflammatory disease [1], and its progression may be affected by local and systemic risk factors, including social and behavioral factors such as smoking and alcohol abuse $[2,3]$. The assessment of risk factors associated with periodontitis is of great importance when determining treatment and prognosis. Many clinical studies have evaluated the relationship between alcohol abuse and the risk of periodontal disease and have reported some evidence of an association [4-9]. Based on their meta-analysis from Wang 
Competing interests: The authors have declared that no competing interests exist. et al., (2016) [10] concluded that alcohol consumption was associated with an increased risk of periodontal disease and must be considered a behavioral risk factor.

The consumption of alcohol is a socially accepted habit and is part of most people's lifestyle. However, alcohol abuse is considered by the World Health Organization (WHO, 2014) [11] to be the third leading cause of death worldwide, preceded only by cancer and cardiovascular disease. Alcohol abuse has negative effects on systemic health, with liver cirrhosis being the most common chronic disease resulting from alcohol hepatotoxicity [12]. In addition, alcohol directly affects the immune system, increasing the risk of severe infections and altering the metabolic functions of bone homeostasis. These effects, together with the pathology of periodontitis, can accelerate the alveolar bone resorption process, thereby affecting the inflammatory response mechanism of periodontitis [13-17].

The deleterious effects of chronic alcohol consumption have been suggested to be dose dependent [6, 8]. In a clinical study, Tezal et al., (2004) [6] observed a significant relationship between the number of drinks per week $(5,10,15$ or 20$)$ and clinical attachment loss (CAL). Lages et al., (2012) [8] observed a higher incidence of periodontitis that corresponded to the frequency of alcohol consumption. According to their results, the prevalence of periodontitis was $53 \%$ in alcohol-dependent individuals, and prevalence of $17.2 \%, 24.0 \%$ and $29.6 \%$ were observed in occasional, moderate and intense alcohol users, respectively. In a recent study with data from the National Health and Nutrition Examination Survey (NHANES, 20092012), Gay et al., (2018) [18] concluded that alcohol consumption was associated with an increased chance of having periodontitis. However, consumption of $<1$ drink per week was associated with similar odds of having periodontitis compared with non-alcohol consumption. However, the exact dose required to affect the pathology of periodontitis remains unclear.

The mechanisms by which chronic alcohol consumption affects the progression of periodontal disease require further investigation. With respect to bone tissue, studies have shown that chronic alcohol consumption can affect bone density, calcium and phosphorus levels [19, 20] and inhibit bone repair [21]. Dal Fabbro et al., (2019) [22], in a recent study concluded that chronic alcohol consumption had a significant effect on the severity of apical periodontitis, exacerbating the inflammatory response and osteoclastogenesis. Consequently, such events resulting from chronic alcohol consumption can compromise the stability of periodontal tissue [6, 8, 23-26]. Moreover, in a study using animal models, Liberman et al., (2011) [27] concluded that ingestion of alcohol at low concentrations (5\%) did not affect alveolar bone loss in induced periodontal disease, suggesting that the adverse effects of alcohol are related to its concentration. For this reason, further experimental studies are needed to clarify the way in which chronic alcohol consumption can alter the pathogenesis of periodontal disease.

No information is available on the association between chronic alcohol consumption and its dose-dependent effects on the progression of periodontitis in rats, so the aim of this study was to evaluate the effects of chronic alcohol consumption at different concentrations on the progression of experimental periodontitis (EP). Thus, the hypothesis tested in this study was that chronic alcohol consumption would increase the severity of EP regardless of the concentration consumed.

\section{Materials and methods}

\subsection{Animals}

A total of 160 male Wistar rats weighing between $250 \mathrm{~g}$ and $300 \mathrm{~g}$ were used. The experimental protocol was approved by the Institutional Ethics Committee on Animal Use and the National Council for Animal Experimentation Control (Concea) (Protocol nº 00636-2013). This study 
was conducted in accordance with ARRIVE (Animal Research: Reporting of In Vivo Experiments) [28].

According to a table generated by a computer program, the animals were divided into the following groups: an EP-NT group $(n=40)$, normal rats with EP; an EP-A14 group $(n=40)$, rats with EP that were exposed to alcohol at a concentration of 14\% (v/v); an EP-A25 group $(\mathrm{n}=40)$, rats with EP that were exposed to alcohol at a concentration of $25 \%(\mathrm{v} / \mathrm{v})$; and an EP-A36 group $(n=40)$, rats with EP that were exposed to alcohol at a concentration of $36 \%$ $(\mathrm{v} / \mathrm{v})$.

\subsection{Administration of alcohol}

The administration of the alcohol solution was initiated 30 days before the induction of EP, and the animals of the EP-A $14^{\circ}, \mathrm{EP}-\mathrm{A} 25^{\circ}$ and EP-A36 groups received $14 \%, 25 \%$ and $36 \% \mathrm{v} /$ $\mathrm{v}$ alcohol solutions, respectively, following the method of D'Souza El-Guindy et al., (2010) [29]. All animals were allowed free access to rodent chow and a single bottle containing the liquid correspondent to the experimental group. Group EP-NT (control animals) were allowed free access to drinking water. Group EP-A14 ${ }^{\circ}$ were allowed free access to drinking $14 \%(\mathrm{v} / \mathrm{v})$ alcohol solution. Group EP-A25 ${ }^{\circ}$ were allowed free access to drinking $25 \%(\mathrm{v} / \mathrm{v})$ alcohol solution. Group EP-A36 were allowed free access to drinking 36\% (v/v) alcohol solution. The animals from test groups were not allowed access to drinking water. The alcohol administration was continued until the end of the experiment.

\subsection{EP induction method and blood sample collection}

To induce EP, sedation and general anesthesia was stated by the combination of $70 \mathrm{mg} / \mathrm{kg}$ of ketamine hydrochloride (Vetaset, Zoetis Iowa, USA) and $6 \mathrm{mg} / \mathrm{kg}$ of xylazine hydrochloride (Coopazine, Coopers São Paulo, Brazil), intramuscularly. Then, \#24 cotton ligatures (Corrente Algodão $n^{\circ} 24$, Coats Corrente, SP, Brazil) were wrapped around the first lower left molars and secured at the gingival sulcus with surgical knots until the end of the experiment [30]. Blood samples were collected for analysis of the liver enzymes alanine aminotransferase (ALT) and aspartate aminotransferase (AST). The blood samples $(2.0 \mathrm{~mL})$ were obtained via cardiac puncture 30 days before ligature placement, on the day of the ligature placement and at the time of euthanasia. In order to assure animal welfare, $2.5 \mathrm{mg} / \mathrm{kg}$ of morphine i.m. was administered daily in the following 3 days after EP induction. Ten animals for each group were euthanized by a lethal dose sodium thiopental of $150 \mathrm{mg} / \mathrm{kg}$ (Thiopentax, Cristália, São Paulo, Brazil) at three, seven, fifteen and thirty days after EP induction.

\subsection{Histological processing and immunohistochemistry}

The specimens were demineralized in 10\% ethylenediaminetetraacetic acid (EDTA) and processed in a conventional manner. Semi-serial sections $(4 \mu \mathrm{m})$ were obtained in the mesiodistal direction, and 5 equidistant sections of each specimen were stained with hematoxylin and eosin (H\&E) for histological and histometric analyses. Other sections were subjected to indirect immunoperoxidase staining with the following primary antibodies: anti-tartrate-resistant acid phosphatase (anti-TRAP).(SC-30833, Santa Cruz Biotechnology, Santa Cruz, CA, USA), anti-receptor activator of nuclear factor kappa-B ligand (anti-RANKL) (SC-7628, Santa Cruz Biotechnology, Santa Cruz, CA, USA) and anti-osteoprotegerin (anti-OPG) (SC-8468, Santa Cruz Biotechnology, Santa Cruz, CA, US). The immunohistochemical processing followed the protocol described by Garcia et al., (2013) [31]. 


\subsection{Analysis of results}

Examiners who were blinded to the treatments performed the analyses.

\subsection{Analysis of ALT and AST}

Serum ALT and AST levels were assessed to evaluate liver abnormalities resulting from chronic alcohol consumption using a kinetic method according to the manufacturer's recommendations (Labtest $\AA$, Lagoa Santa-MG, Brazil). The samples were analyzed using a LabQuest semi-automatic biochemistry analyzer (Bioplus, Barueri -SP, Brazil).

\subsection{Histological analysis}

A certified histologist (EE) evaluated the following histological parameters according to the protocol proposed by Almeida et al., (2015) [32]: 1) the nature and degree of inflammation; 2) the extent of the inflammatory process; 3 ) the presence and extent of tissue necrosis; 4) the presence, extent and nature of bone, cementum and dentin resorption; 5) the status of the vasculature; 6) the structural pattern of the extracellular matrix of the periodontal tissue; and 7) the cellularity pattern of the periodontal tissue.

\subsection{Histometric analysis of percentage bone in the furcation region (PBF)}

Another examiner (VFCP) performed the histometric analysis. An image analysis system (Axiovision 4.8.2, Carl Zeiss MicroImaging GmbH, 07740 Jena, Germany) was used to measure (in square millimeters) the total furcation area, the bone area in the furcation region and the PBF (calculated according to the method of Garcia et al. (2015) [33].

\subsection{Immunohistochemical analyses}

A certified histologist (EE) performed the immunohistochemical analyses. A semi-quantitative analysis of RANKL and OPG immunoreactivity pattern in the furcation area was performed with 400x magnification. Three histological sections were used for each animal, and the following immunostaining criteria were used based on the protocol described by Garcia et al., (2015) [33]: 0 , total absence of immunoreactivity pattern (IR) cells; 1 , low immunoreactivity pattern (approximately 25\% IR cells); 2, moderate immunoreactivity pattern (approximately 50\% IR cells); and 3, high immunoreactivity pattern (approximately 75\% IR cells). A quantitative analysis was performed for TRAP using 5 sections from each animal. The TRAP+ multinucleated cells per square millimeter were counted in a $1000 \times 1000 \mu \mathrm{m}$ area at the center of the interradicular septum at 200x magnification. The boundary of this area was the outer contour of the alveolar bone tissue in the furcation region extending apically to $1000 \mu \mathrm{m}$.

\subsection{Examiner calibration}

All the above-identified examiners were blinded to the experimental groups prior to histometric and immunohistochemical analyses. The examiners were trained, and the PBF and TRAP+ cell measurements were carried out in duplicate. The paired t-test was used to calculate the intraexaminer error. A $\mathrm{P}$ value $>0.05$ in the paired $\mathrm{t}$-test was used to estimate the feasibility of the proposed method.

\subsection{Statistical analysis}

With a sample size of $10(\mathrm{P}=0.05)$, the study power was $89 \%$. The collected data were analyzed using software (BioStat version 5.0, Belém, PA, Brazil). The normality of the histometric and 
immunohistochemical data was verified using the Shapiro-Wilk test $(\mathrm{P} \leq 0.05)$. When a significant difference was indicated by analysis of variance (ANOVA), multiple comparisons were performed using the Tukey test $(\mathrm{P} \leq 0.05)$.

\section{Results}

\subsection{Analysis of ALT and AST}

The ALT results are shown in Fig 1A. In the intragroup analysis, the EP-A14, EP-A25, and EP-A36 groups showed higher levels of ALT on days 0, 3, 7, 15 and 30 compared to baseline $(\mathrm{p} \leq 0.05)$. EP-A14 and EP-A25 groups showed higher levels of ALT on days 7, 15 and 30 compared to 0 day $(\mathrm{p} \leq 0.05)$. EP-A36 group showed higher levels of ALT on days 3, 7, 15 and 30 compared to 0 day $(\mathrm{p} \leq 0.05)$.

The AST results are shown in Fig 1B. In the intragroup analysis, the EP-NT group showed significant differences, with higher AST levels on days 3, 7, 15 and 30 compared to baseline and day 0 in the same experimental group $(\mathrm{p} \leq 0.05)$. The EP-A14, EP-A25 and EP-A36 groups showed higher levels of AST at day 0 compared to baseline $(\mathrm{p} \leq 0.05)$. At days 3, 7, 15 and 30, the EP-A14, EP-A25 and EP-A36 groups showed higher levels of AST compared to baseline and day 0 of their respective experimental groups $(\mathrm{p} \leq 0.05)$.

\subsection{Histological analysis}

The histological analysis results are shown in Fig 2. Three days after ligature placement, all the experimental groups exhibited severe disorganization of the periodontal ligament in the furcation region. An intense inflammatory infiltrate composed mainly of polymorphonuclear neutrophils was present in this region, and the magnitude of the local inflammatory response was
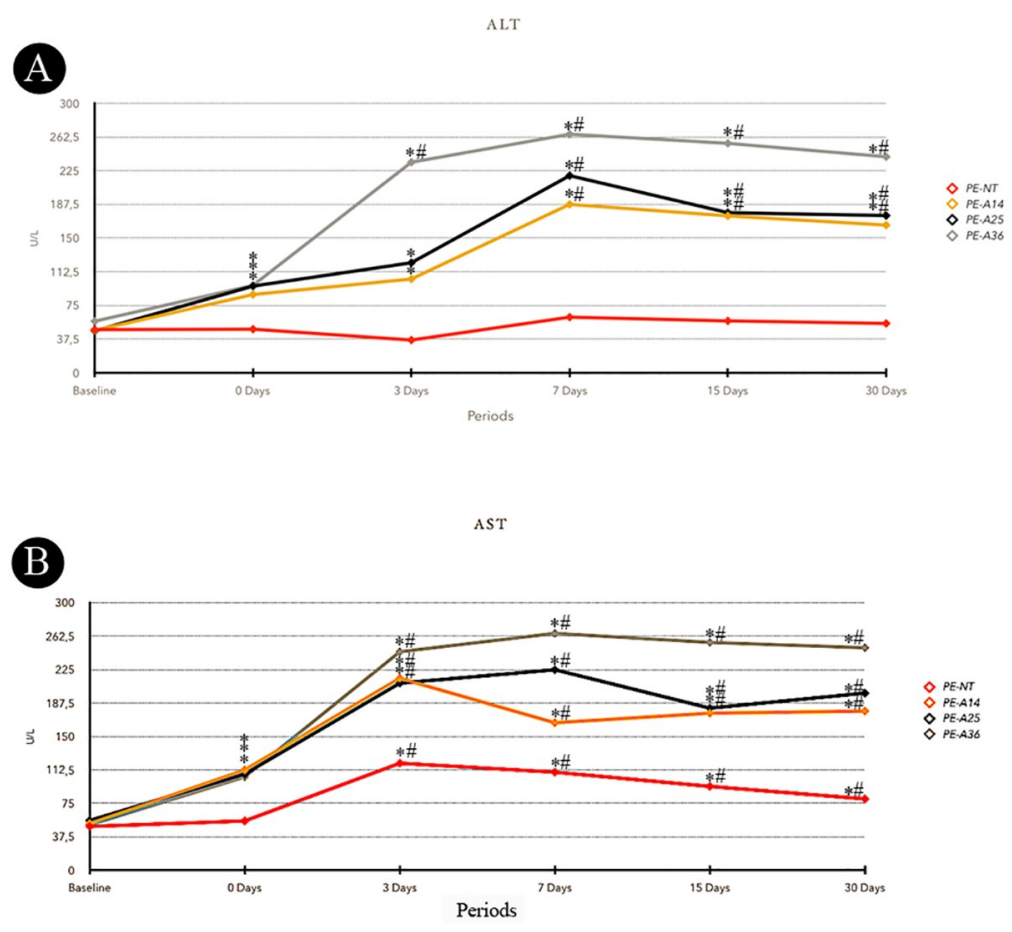

Fig 1. A- Means of ALT serum levels for each group and period. B- Means of AST serum levels for each group and period. Symbols: * statistically significant difference with base line period at the same group. \#statistically significant difference with 0 day period at the same group. ANOVA and Tukey test $(\mathrm{p} \leq 0.05)$.

https://doi.org/10.1371/journal.pone.0232731.g001 

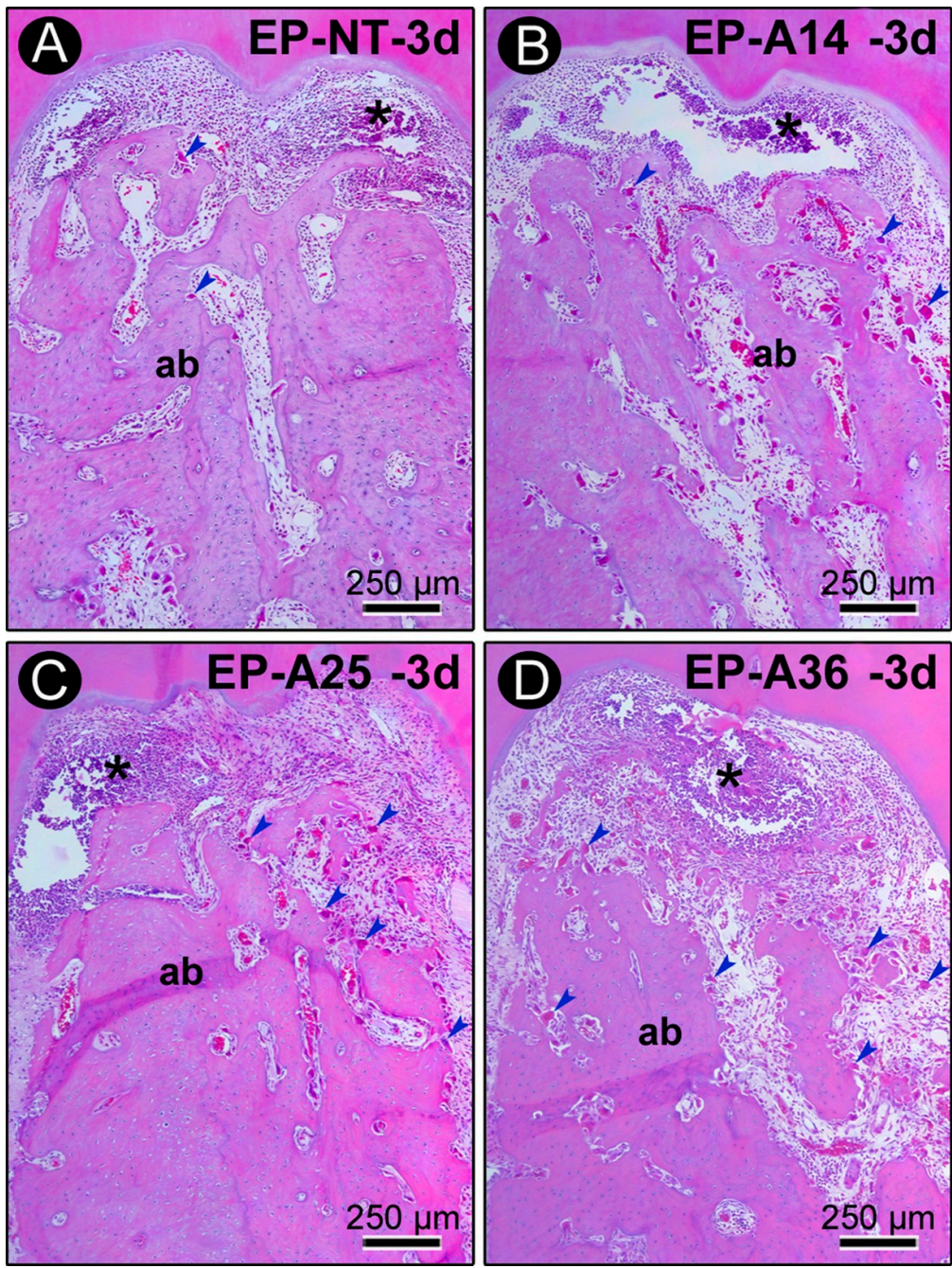

Fig 2. Histopathological characteristics of the furcation region of the mandibular first molars of rats with PE in groups EP (A), EP-A14 (B), EP-A25 (C) and EP-A36 (D) 3 days after ligature placement. Photomicrographs showing the inflammatory infiltrate. Abbreviations and symbols: ab, alveolar bone; ${ }^{*}$, inflammatory infiltrate. Staining: hematoxylin and eosin (HE). Magnification: 100x. Scale bar: A-d: $250 \mu \mathrm{m}$.

https://doi.org/10.1371/journal.pone.0232731.g002

much higher in the groups treated with alcohol. At this time point, a great number of osteoclasts could be observed that were distributed in the furcation region of the alveolar bone, especially in the alveolar crest, which had active bone resorption areas. The levels of bone resorption were similar in all of the experimental groups. However, osteoclast recruitment to the region was significantly increased in the groups treated with alcohol.

Further results are shown in Fig 3. Seven days after ligature placement, tissue disorganization was prevalent in the furcation region, with significantly compromised connective tissue and alveolar bone tissue. Inflammatory infiltration was more extensive, and this inflammatory response was much more exaggerated in the groups treated with alcohol, especially in the EP-A25 and EP-A36 groups. Active bone resorption was present in all specimens from all experimental groups. During this period, the groups treated with alcohol had interradicular septa that consisted of very irregular, extremely thin bone trabeculae with large medullary spaces. In groups EP-A25 and EP-A36, these medullary spaces contained large concentrations of inflammatory cells. 

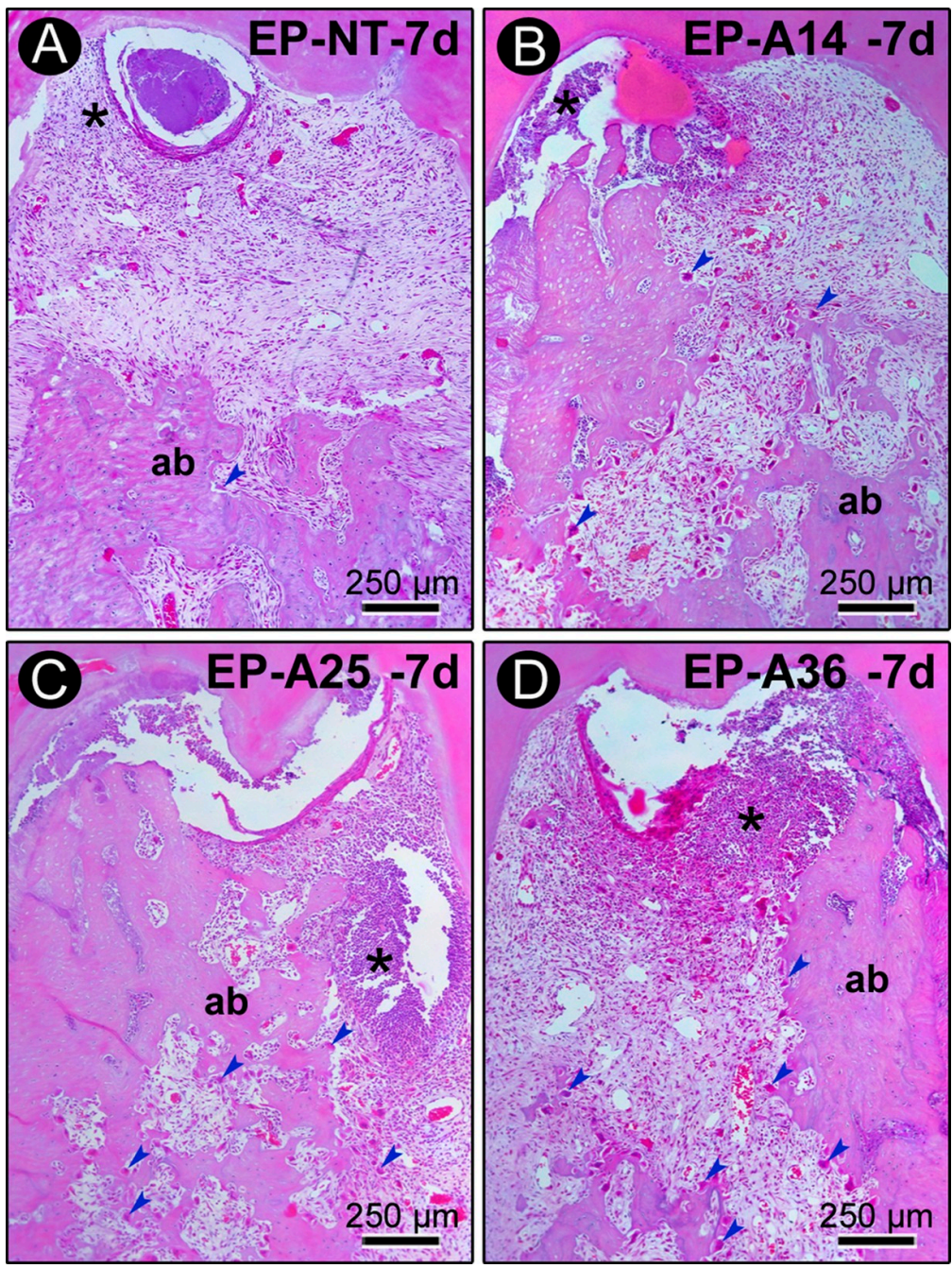

Fig 3. Histopathological characteristics of the furcation region of the mandibular first molars of rats with PE in groups EP (A), EP-A14 (B), EP-A25 (C) and EP-A36 (D) 7 days after ligature placement. Photomicrographs showing the inflammatory infiltrate and alveolar bone loss. Abbreviations and symbols: ab, alveolar bone; *, inflammatory infiltrate. Staining: hematoxylin and eosin (HE). Magnification: 100x. Scale bar: A-d: $250 \mu \mathrm{m}$.

https://doi.org/10.1371/journal.pone.0232731.g003

At days 15 and 30 after ligature placement, chronic inflammatory infiltrate was present in the connective tissue of the furcation region of the EP-NT group, and the alveolar bone loss had stabilized. At day 15 in the groups treated with alcohol, the inflammatory response and bone resorption activity were still very intense. In groups EP-A14 and EP-A25, a mild inflammatory response was observed only after 30 days (Fig 4). During this period, these groups still had areas of active bone resorption. In the EP-A36 group, the local inflammatory response and bone resorption activity were still exacerbated at 30 days compared with the other groups.

\subsection{Histometric analysis}

The Kappa test indicated a level of intraexaminer agreement for the PBF measurements of $94 \%$, which was a high level of agreement.

The results are shown in Table 1. According to the intragroup analysis comparing the different periods, the EP-A14, EP-A25 and EP-A36 groups had lower PBFs at days 7, 15 and 30 

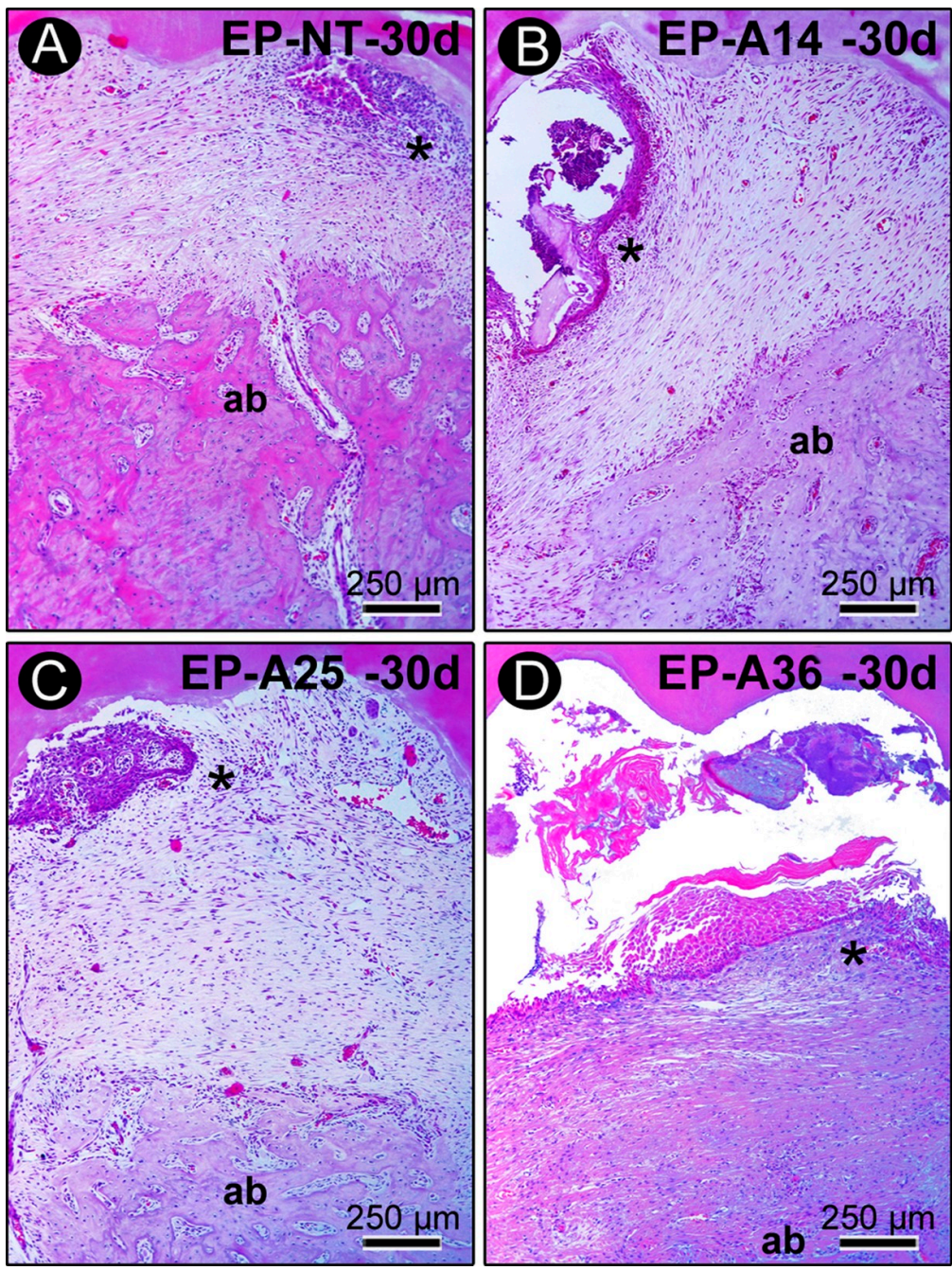

Fig 4. Histopathological characteristics of the furcation region of the mandibular first molars of rats with $\mathrm{PE}$ in groups EP (A), EP-A14 (B), EP-A25 (C) and EP-A36 (D) 30 days after ligature placement. Photomicrographs showing the inflammatory infiltrate and alveolar bone loss. Abbreviations and symbols: ab, alveolar bone; *, inflammatory infiltrate. Staining: hematoxylin and eosin (HE). Magnification: 100x. Scale bar: A-d: $250 \mu \mathrm{m}$.

https://doi.org/10.1371/journal.pone.0232731.g004

Table 1. Means and standard deviations $(M \pm S D)$ of the histometric data for PBF (\%) in the furcation regions of the left mandibular first molars according to groups and periods.

\begin{tabular}{|c|c|c|c|c|}
\hline & $\mathbf{3}$ days & $\mathbf{7}$ days & $\mathbf{1 5}$ days & $74.05 \pm 5.07$ \\
\hline EP-NT & $77.98 \pm 2.54$ & $77.12 \pm 6.23$ & $74.05 \pm 6.29$ & $51.51 \pm 7.67^{*, \dagger}$ \\
\hline EP-A14 & $77.54 \pm 5.99$ & $57.45 \pm 9.24^{*, \dagger}$ & $48.62 \pm 7.30^{*, \dagger}$ & $50.91 \pm 11.15^{*, \dagger}$ \\
\hline EP-A25 & $75.81 \pm 4.67$ & $55.16 \pm 6.92^{*, \dagger}$ & $51.48 \pm 8.42^{*, \dagger}$ & $34.50 \pm 9.65^{*, \dagger}$ \\
\hline
\end{tabular}

Symbols:

*, statistically significant difference with 3 days in the same group.

${ }^{\dagger}$, statistically significant difference with group EP-NT at the same time point. 
compared with the values obtained at day $3(\mathrm{P} \leq 0.05)$. According to the intergroup analysis comparing the different groups, the animals of groups EP-A14, EP-A25 and EP-A36 had lower PBFs than the animals belonging to the EP-NT group at days 7, 15 and $30(\mathrm{P} \leq 0.05)$. At day 3 , there were no significant differences between the groups, and there were no significant differences between the EP-A14, EP-A25 and EP-A36 groups at any time point.

\subsection{Immunohistochemical analysis}

The RANKL and OPG results are shown in Fig 5. For RANKL, the EP-NT group exhibited high immunoreactivity pattern at days 3 and 7 and moderate immunoreactivity pattern at days 15 and 30. The EP-A14 group exhibited high immunoreactivity pattern at days 3, 7 and 15 and moderate immunoreactivity pattern at day 30 . The EP-A25 and EP-A36 groups exhibited high immunoreactivity pattern at all time points. The OPG immunoreactivity pattern was predominantly low at all time points in all the groups.

The TRAP results are shown in Table 2 and Fig 5. According to the intergroup analysis comparing the different experimental groups, the EP-A14, EP-A25 and EP-A36 groups had a higher number of TRAP cells than the EP-NT group at all time points $(\mathrm{P} \leq 0.05)$. The EP-A25 group had a higher number of TRAP cells than the EP-A14 group at day 7, and the EP-A36 group had a higher number of TRAP cells than the EP-A14 and EP-A25 groups at all time points $(\mathrm{P} \leq 0.05)$.

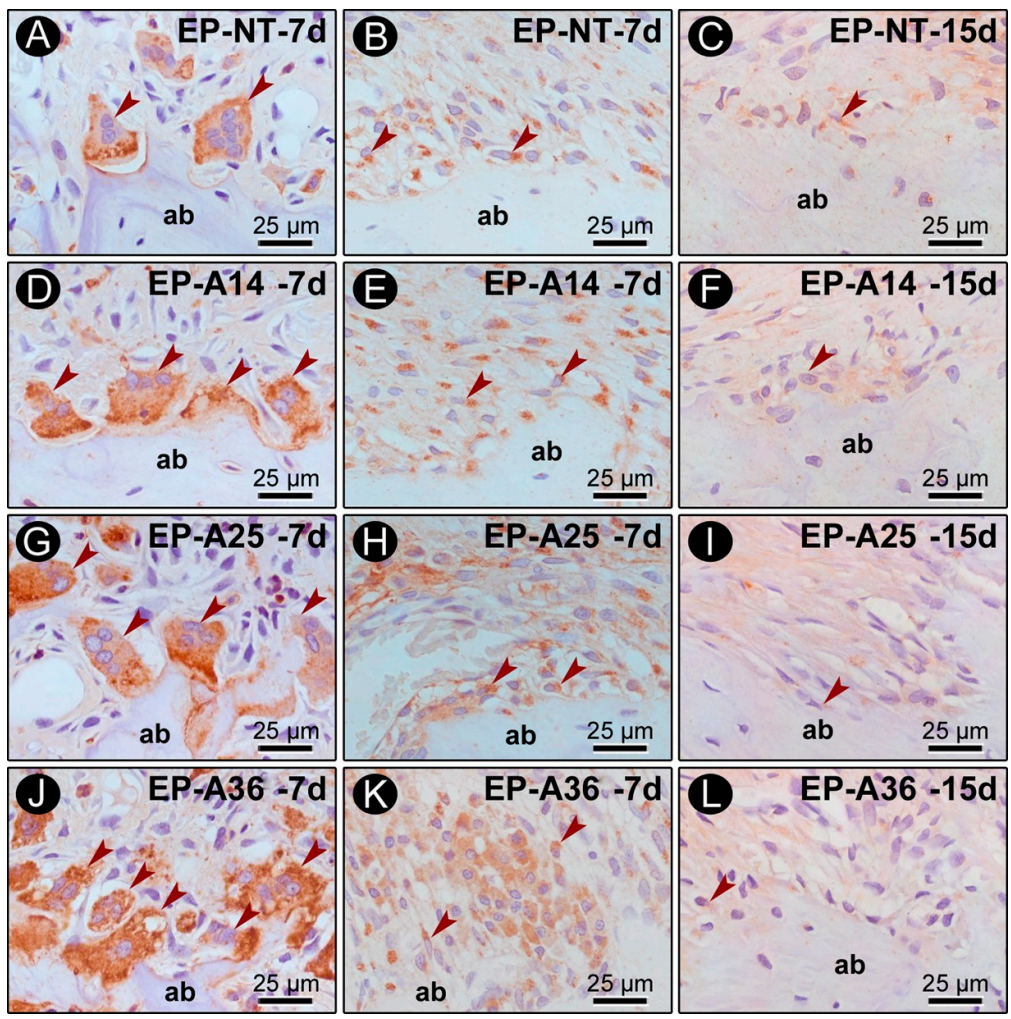

Fig 5. TRAP, RANKL and OPG immunolabeling in the furcation region of the mandibular first molar. Photomicrographs showing the immunolabeling pattern of TRAP (A, D, G, J), RANKL (B, E, H, K) at 7 days after ligature placement, and OPG (C, F, I, L) at 15 days after ligature placement. Abbreviations: ab, alveolar bone; arrows, immunorective cells. Magnification: 1000x. Scale bars: $25 \mu \mathrm{m}$. Counterstaining: hematoxylin.

https://doi.org/10.1371/journal.pone.0232731.g005 
Table 2. Means and standard deviations $(M \pm S D)$ of the amount of TRAP in the furcation regions of the left mandibular first molars according to groups and periods.

\begin{tabular}{|c|c|c|c|c|}
\hline & $\mathbf{3}$ days & $\mathbf{7}$ days & $\mathbf{1 5}$ days & $\mathbf{3 0}$ days \\
\hline EP-NT & $10.94 \pm 4.0$ & $18.20 \pm 9.77$ & $9.70 \pm 3.67$ & $3.94 \pm 3.5$ \\
\hline EP-A14 & $30.88 \pm 6.81^{\dagger, 9}$ & $31.60 \pm 1.99^{\dagger, 9}$ & $34.58 \pm 6.82^{\dagger, 9}$ & $38.98 \pm 4.56^{\dagger, 9}$ \\
\hline EP-A25 & $31.76 \pm 6.94^{\dagger, 9}$ & $38.40 \pm 5.15^{\dagger, 8,9}$ & $31.80 \pm 2.48^{\dagger, 9}$ & $35.04 \pm 4.84^{\dagger, 9}$ \\
\hline EP-A36 & $57.64 \pm 4.77^{\dagger}$ & $50.60 \pm 6.27^{\dagger}$ & $54.46 \pm 5.91^{\dagger}$ & $59.00 \pm 3.64^{\dagger}$ \\
\hline
\end{tabular}

Symbols:

${ }^{\dagger}$, statistically significant difference with group EP-NT at the same time point.

\&, statistically significant difference with group EP-A14 at the same time point.

9. statistically significant difference with group EP-A36 at the same time point. ANOVA and Tukey test $(\mathrm{p} \leq 0.05)$.

https://doi.org/10.1371/journal.pone.0232731.t002

\section{Discussion}

The hypothesis that chronic alcohol consumption would increase the severity of EP regardless of the concentration consumed was confirmed in our study. This study demonstrate that chronic alcohol consumption increases the severity of EP in a dose-dependent manner by increasing the magnitude of the local inflammatory response, RANKL immunoreactivity pattern and the number of TRAP+ cells, which leads to greater alveolar bone loss and a lower PBF.

Various methodologies, which differed in regard to the way the alcohol was administered, the concentration of the alcohol solution, the age of the animals and the type of alcohol, have been applied to evaluate the effects of alcohol on EP [27, 34-37]. Consequently, the lack of methodological standardization has given rise to conflicting results. For this reason, the animals in the present study were exposed to different alcohol concentrations (14\%, 25\% and $36 \%$ ) to determine which concentration could demonstrate a positive association between alcohol consumption and periodontal disease.

The experimental model of chronic alcoholism used in this study was proposed by Souza et al., (2009) [34]. A 30-day period of prior exposure to alcohol in rats mimics the behavior of a chronic alcoholic [34] and has been proven to be an effective experimental model of alcoholism $[38,39]$. This model is easy to implement and extremely practical, especially when long experimental periods are required. It also involves virtually no manipulation of the animal, as is often required in other experimental models. In this study, the serum ALT and AST levels confirmed the liver damage caused by chronic alcohol consumption in the groups exposed to alcohol, as changes in liver enzymes have been observed in alcoholic patients [40].

The effects of alcohol on immune responses depend on the timing of consumption. In acute consumption, the anti-inflammatory effects are due to a reduction in pro-inflammatory cytokines and an increase in anti-inflammatory cytokines, which suggests a protective effect [41]. In chronic use, alcohol acts in an opposite way by increasing the levels of pro-inflammatory cytokines, such as tumor necrosis factor (TNF), interleukin 1 (IL-1) and interleukin 6 (IL6), and reducing the levels of anti-inflammatory cytokines [17, 42]. In our results, the inflammatory response in PE was more exacerbated in alcohol dose-dependent manner, corroborating these studies.

The results of the present study revealed that in the EP-NT group, comprising animals that were not exposed to alcohol, there was a gradual increase in inflammatory infiltrate 3 and 7 days after ligature placement and a subsequent reduction in its magnitude as the inflammatory process progressed as well as a concomitant reduction in the $\mathrm{PBF}$. These results agree with previous studies $[43,44]$. Similarly, the EP-A14, EP-A25 and EP-A36 groups, which consisted of 
animals that were exposed to alcohol, exhibited similar histopathological characteristics. However, the magnitude of the local inflammatory responses was much higher, especially in the groups that were exposed to higher alcohol concentrations, and these groups also had large areas of bone necrosis.

There was a gradual reduction in the PBF over time in all the groups that were exposed to alcohol. However, there were no significant differences among the groups of animals that were exposed to alcohol. Such histological and histometric findings demonstrate that chronic alcohol use greatly exacerbates the severity of EP, irrespective of the concentration was used. In contrast to our results, a study by Liberman et al., (2011) [27] revealed that alcohol intake did not affect alveolar bone loss in EP. However, the concentration used in their study (5\%) is considered low and was below the lowest concentration used in our study (14\%). Our data corroborate previous studies that used concentrations ranging from $10 \%$ to $30 \%$ and found that chronic alcohol consumption caused alveolar bone loss and decreased bone density in animals with EP [35, 45, 46].

Periodontal bone remodeling is locally regulated by the RANK-RANKL-OPG system, which modulates alveolar bone resorption. RANK, its ligand RANKL and OPG are included in this system [47]. Thus, the resorptive activity of alveolar bone in this study was evaluated by using immunohistochemistry to detect the 2 key regulators of local bone metabolism, RANKL and OPG, and by quantifying the TRAP+ multinucleated cells. Previous experimental studies have evaluated the effects of alcohol consumption on bone metabolism and osteoclastogenesis and noted a decrease in osteoblast activity [48] and an increase in osteoclastic activity [49]. In the present study, the OPG immunostaining was consistently low in all the experimental groups and at all the time points. In contrast, RANKL immunoreactivity pattern showed a dose-dependent increase when compared to the EP-NT group, especially 3 and 7 days after ligature placement. Corroborating the OPG and RANKL results, we observed that the groups exposed to chronic alcohol had higher numbers of TRAP+ multinucleated cells in the furcation region than the EP-NT group. The EP-A36 group had a higher number of TRAP+ cells than the EP-A14 and EP-A25 groups. The same result was found in a recent study that evaluated apical periodontitis in animals with chronic alcohol consumption at a concentration of $20^{\circ}$ [22]. These results therefore suggest that the regulation of osteoclastogenesis in animals subjected to chronic alcohol consumption is dose dependent.

The way in which chronic alcohol consumption stimulates osteoclastogenesis has been studied by Iitsuka et al., (2012) [48]. They concluded that alcohol stimulates osteoclastogenesis by increasing RANK expression, which is mediated by the production of reactive oxygen species and the activation of extracellular signal-regulated kinases in precursor osteoclast cells and results in an increase in RANKL in osteoblasts. In the pathology of periodontal disease, a series of cascading events leads to osteoclastogenesis, which is regulated by the RANK-RANKL-OPG system $[32,47]$. We can therefore infer that chronic alcohol consumption can worsen the severity of periodontal disease. In this context, the results of our study demonstrate that chronic alcohol consumption can worsen alveolar bone loss in EP via RANKL upregulation. In contrast to our results, Bastos et al. [35] observed a significantly lower number of TRAP + cells, as well as an increase in OPG, in a group with induced EP that was exposed to alcohol compared with the control group. However, an increase in RANKL was observed in the same animals with EP that were exposed to chronic alcohol consumption when compared with the control group. It is important to note that in the same study [35], increased bone loss and decreased bone density were observed in animals with EP that were exposed to chronic alcohol consumption, confirming the deleterious effects of chronic alcohol consumption on the resorptive activity of alveolar bone.

The histological and immunohistochemical findings of our study indicate that chronic alcohol consumption has dose-dependent effects. In the animal groups that were exposed to 
alcohol, higher concentrations resulted in more severe inflammation, increased RANKL immunoreactivity and a significantly higher number of TRAP+ cells. However, the magnitude of the immunoinflammatory responses did not result in statistically significant changes in the PBF among the animal groups that were exposed to alcohol, although it may have had significant effects on bone tissue in later periods. It is important to note that pre-clinical studies using animals are necessary to protect human health and guide clinical studies investigating therapeutic strategies for the treatment of periodontal disease.

\section{Conclusion}

Within the limits of the present study, we can conclude that chronic alcohol consumption at different concentrations increases the severity of EP regardless of the concentration consumed in a dose-dependent manner by increasing the magnitude of the local inflammatory response and stimulating alveolar bone resorption.

\section{Supporting information}

S1 File. Data set for the ALT and AST assessments.

S2 File. Data set for the histometric analysis of the PBF. (PDF)

S3 File. Data set for the immunohistochemical analysis of TRAP. (PDF)

\section{Author Contributions}

Conceptualization: Juliano Milanezi de Almeida, Edilson Ervolino.

Data curation: Juliano Milanezi de Almeida, Victor Fabrizio Cabrera Pazmino, Vivian Cristina Noronha Novaes, Maria José Hitomi Nagata.

Formal analysis: Suely Regina Mogami Bomfim, Edilson Ervolino.

Funding acquisition: Juliano Milanezi de Almeida, Victor Fabrizio Cabrera Pazmino.

Investigation: Suely Regina Mogami Bomfim, Fred Lucas Pinto Oliveira, Edilson Ervolino.

Methodology: Juliano Milanezi de Almeida.

Project administration: Juliano Milanezi de Almeida, Victor Fabrizio Cabrera Pazmino, Vivian Cristina Noronha Novaes.

Resources: Fred Lucas Pinto Oliveira.

Supervision: Suely Regina Mogami Bomfim.

Validation: Edilson Ervolino.

Visualization: Henrique Rinald Matheus.

Writing - original draft: Victor Fabrizio Cabrera Pazmino.

Writing - review \& editing: Juliano Milanezi de Almeida, Vivian Cristina Noronha Novaes, Maria José Hitomi Nagata, Edilson Ervolino. 


\section{References}

1. Kinane DF. Causation and pathogenesis of periodontal disease. Periodontology 2000. 2001; 25, 8-20. https://doi.org/10.1034/j.1600-0757.2001.22250102.x PMID: 11155179

2. Stanford TW, Rees TD. Acquired immune suppression and other risk factors/indicators for periodontal disease progression. Periodontology 2000. 2003; 32, 118-135. https://doi.org/10.1046/j.0906-6713. 2003.03210.x PMID: 12756038

3. Nunn ME. Understanding the etiology of periodontitis: an overview of periodontal risk factors. Periodontol 2000. 2003; 32, 11-23. https://doi.org/10.1046/j.0906-6713.2002.03202.x PMID: 12756030

4. Sakki TK, Knuuttila ML, Vimpari SS, Hartikainen MS. Association of lifestyle with periodontal health. Community Dent Oral Epidemiol. 1995; 23, 155-158. https://doi.org/10.1111/j.1600-0528.1995. tb00220.x PMID: 7634770

5. Tezal M, Grossi SG, Ho AW, Genco RJ. The effect of alcohol consumption on periodontal disease. J Periodontology. 2001; 72, 183-189. https://doi.org/10.1902/jop.2001.72.2.183

6. Tezal M, Grossi SG, Ho AW, Genco RJ. Alcohol consumption and periodontal disease. The third national health and nutrition examination survey. J Clin Periodontol. 2004; 31, 484-488. https://doi.org/ 10.1111/j.1600-051X.2004.00503.x PMID: 15191580

7. Shimazaki Y, Saito T, Kiyohara Y, Kato I, Kubo M, lida M, et al. Relationship between drinking and periodontitis: the Hisayama study. J Periodontology. 2005; 76, 1534-1541. https://doi.org/10.1902/jop. 2005.76.9.1534

8. Lages EJ, Costa FO, Lages EM, Cota LO, Cortelli SC, Nobre-Franco GC, et al. Risk variables in the association between frequency of alcohol consumption and periodontitis. J Clin Periodontol. 2012; 39, 115-122. https://doi.org/10.1111/j.1600-051X.2011.01809.x PMID: 22092940

9. Hach $M$, Holm-Pedersen $P$, Adegboye AR, Avlund $K$. The effect of alcohol consumption on periodontitis in older Danes. Int J Dent Hyg. 2015; 13, 261-267. https://doi.org/10.1111/idh.12121 PMID: 25684316

10. Wang J, Lv J, Wang W, Jiang X. Alcohol consumption and risk of periodontitis: a meta-analysis. J Clin Periodontol. 2016; 43, 572-583. https://doi.org/10.1111/jcpe.12556 PMID: 27029013

11. World Health Organization. Global status report on alcohol and health. http://www.who.int/substance abuse/publications/alcohol_2014/en/ Accessed 21/03/2020.

12. Lieber CS. ALCOHOL: its metabolism and interaction with nutrients. Annu Rev Nutr. 2000; 20, 395430. https://doi.org/10.1146/annurev.nutr.20.1.395 PMID: 10940340

13. Messingham KAN, Faunce DE, Kovacs EJ. Alcohol, injury, and cellular immunity. Alcohol. 2002; 28, 137-149. https://doi.org/10.1016/s0741-8329(02)00278-1 PMID: 12551755

14. Ilich JZ, Brownbill RA, Tamborini L, Crncevic-Orlic Z. To drink or not to drink: how are alcohol, caffeine and past smoking related to bone mineral density in elderly women? J Am Coll Nutr. 2002; 21, 536 544. https://doi.org/10.1080/07315724.2002.10719252 PMID: 12480799

15. Pavia CS, Mothe M, Kavanagh M. Influence of alcohol on antimicrobial immunity. Biomed Pharmacother. 2004; 58, 84-9. https://doi.org/10.1016/j.biopha.2003.12.005 PMID: 14992788

16. Crews FT, Bechara R, Brown LA, Guidot DM, Mandrekar P, Oak S, et al. Cytokines and alcohol. Alcohol Clin Exp Res. 2006; 30:720-730. https://doi.org/10.1111/j.1530-0277.2006.00084.x PMID: 16573591

17. Irie K, Tomofuji T, Tamaki N, Sanbe T, Ekuni D, Azuma T, et al. Effects of ethanol consumption on periodontal inflammation in rats. J Dent Res. 2008; 87, 456-460. https://doi.org/10.1177/ 154405910808700511 PMID: 18434576

18. Gay IC, Tran DT, Paquette DW. Alcohol intake and periodontitis in adults aged $\geq 30$ years: NHANES 2009-2012. J Periodontol. 2018; 89, 625-634. https://doi.org/10.1002/JPER.17-0276 PMID: 29572839

19. Nyquist F, Düppe H, Obrant KJ, Bondeson L, Nordsletten L. Effects of alcohol on bone mineral and mechanical properties of bone in male rats. Alcohol Alcohol. 2002; 37, 21-24. https://doi.org/10.1093/ alcalc/37.1.21 PMID: 11825852

20. Broulík PD, Vondrová J, Růzicka P, Sedlácek R, Zíma T. The effect of chronic alcohol administration on bone mineral content and bone strength in male rats. Physiol Res. 2010; 59, 599-604. PMID: 19929136

21. Chakkalakal DA, Novak JR, Fritz ED, Mollner TJ, McVicker DL, Garvin KL, et al. Inhibition of bone repair in a rat model for chronic and excessive alcohol consumption. Alcohol. 2005; 36, 201-214. https://doi. org/10.1016/j.alcohol.2005.08.001 PMID: 16377462

22. Dal-Fabbro R, Marques-de-Almeida M, Cosme-Silva L, Ervolino E, Cintra LTA, Gomes-Filho JE. Chronic alcohol consumption increases inflammation and osteoclastogenesis in apical periodontitis. Int Endod J. 2019; 52, 329-336. https://doi.org/10.1111/iej.13014 PMID: 30218448 
23. Szabo G. Consequences of alcohol consumption on host defence. Alcohol Alcohol. 1999; 34, 830841. https://doi.org/10.1093/alcalc/34.6.830 PMID: 10659718

24. Torrungruang K, Tamsailom S, Rojanasomsith K, Sutdhibhisal S, Nisapakultorn K, Vanichjakvong O, et al. Risk indicators of periodontal disease in older Thai adults. J. Periodontol. 2005; 76, 558-565. https://doi.org/10.1902/jop.2005.76.4.558 PMID: 15857096

25. Kongstad J, Hvidtfeldt UA, Grønbæk M, Jontell M, Stoltze K, Holmstrup P. Amount and type of alcohol and periodontitis in the Copenhagen City Health Study. J Clin Periodontol. 2008; 35, 1031-1039. https://doi.org/10.1111/j.1600-051X.2008.01325.x.

26. Tomofuji T, Sanbe T, Ekuni D, Azuma T, Irie K, Maruyama T, et al. Oxidative damage of rat liver induced by ligature-induced periodontitis and chronic ethanol consumption. Arch Oral Biol. 2008; 53, 11131118. https://doi.org/10.1016/j.archoralbio.2008.05.015 PMID: 18603227

27. Liberman D.N., Pilau R.M., Gaio E.J., Orlandini L.F., Rösing C.K., 2011. Low concentration alcohol intake may inhibit spontaneous alveolar bone loss in Wistar rats. Arch Oral Biol. 56, 109-113. https:// doi.org/10.1016/j.archoralbio.2010.09.012 PMID: 20943212

28. Kilkenny C, Browne W, Cuthill IC, Emerson M, Alman DG. NC3Rs Reporting Guidelines Working Group. Animal research: reporting in vivo experiments: the ARRIVE guidelines. J Gene Med. 2010; 12, 561-563. https://doi.org/10.1002/jgm.1473 PMID: 20607692

29. D'Souza El-Guindy NB, Kovacs EJ, De Witte P, Spies C, Littleton JM, de Villiers WJ, et al. Laboratory models available to study alcohol-induced organ damage and immune variations: choosing the appropriate model. Alcohol Clin Exp Res. 2010; 34, 1489-1511. https://doi.org/10.1111/j.1530-0277.2010. 01234.x PMID: 20586763

30. De Almeida JM, Theodoro LH, Bosco AF, Nagata MJ, Oshiiwa M, Garcia VG. Influence of photodynamic therapy on the development of ligature-induced periodontitis in rats. J Periodontol. 2007; 78:566-75. https://doi.org/10.1902/jop.2007.060214 PMID: 17335382

31. Garcia VG, Gualberto Júnior EC, Fernandes LA, Bosco AF, Hitomi Nagata MJ, Casatti CA, et al Adjunctive antimicrobial photodynamic treatment of experimentally induced periodontitis in rats with ovariectomy. J Periodontol. 2013; 84, 556-565. https://doi.org/10.1902/jop.2012.120163 PMID: 22680299

32. De Almeida J, Ervolino E, Bonfietti LH, Novaes VC, Theodoro LH, Fernandes LA, et al. Adjuvant Therapy with Sodium Alendronate for the Treatment of Experimental Periodontitis in Rats. J Periodontol. 2015; 86, 1166-1175. https://doi.org/10.1902/jop.2015.150166 PMID: 26062841

33. Garcia VG, Novaes VC, de Almeida JM, Longo M, Ervolino E, Bomfim SR, et al. Evaluation of the progression and treatment of experimental periodontitis in rats subjected to chemotherapy with 5-fluorouracil. Support Care Cancer. 2015; 23, 2007-2017. https://doi.org/10.1007/s00520-014-2563-y PMID: 25519757

34. Souza DM, Ricardo LH, Kantoski KZ, Rocha RF. Influence of alcohol consumption on alveolar bone level associated with ligature-induced periodontitis in rats. Braz Oral Res. 2009; 23, 326-332. https:// doi.org/10.1590/s1806-83242009000300017 PMID: 19893970

35. Bastos MF, Gaag GL, Romero JR, Gabrili JJ, Marques MR, Duarte PM. Effects of Cachaça, a typical Brazilian alcoholic beverage, on alveolar bone loss and density: a study in peripubertal rats. Arch Oral Biol. 2011; 56, 109-13. https://doi.org/10.1016/j.archoralbio.2010.09.012

36. Bannach SV, Teixeira FB, Fernandes LM, Ferreira RO, Santana LN, Fontes-Júnior EA, et al. Alveolar bone loss induced by chronic ethanol consumption from adolescence to adulthood in Wistar rats. Indian J Exp Biol. 2015; 53, 93-7. PMID: 25757239

37. Surkin PN, Ossola CÁ, Mohn CE, Elverdin JC, Fernández-Solari J. Chronic alcohol consumption alters periodontal health in rats. Alcohol Clin Exp Res. 2014; 38, 2001-2007. https://doi.org/10.1111/acer. 12436 PMID: 24931716

38. Turner RT, Kidder LS, Kennedy A, Evans GL, Sibonga JD. Moderate alcohol consumption suppresses bone turnover in adult female rats. J J Bone Miner Res. 2001; 16, 589-594. https://doi.org/10.1359/ jbmr.2001.16.3.589

39. Kim HJ, Choi YS, Jeong MJ, Kim BO, Lim SH, Kim DK, et al. Expression of UNCL during development of periodontal tissue and response of periodontal ligament fibroblasts to mechanical stress in vivo and in vitro. Cell Tissue Res. 2007; 327, 25-31. https://doi.org/10.1007/s00441-006-0304-3 PMID: 17004066

40. Hietela J, Koivisto H, Anttila P, Niemela O. Comparison of the combined marker GGT-CDT and the conventional laboratory markers of alcohol abuse in heavy drinkers, moderate drinkers and abstainers. Alcohol Alcohol. 2006; 41, 528-533. https://doi.org/10.1093/alcalc/agl050 PMID: 16799164

41. Fu P, Peng C, Ding JY, Asmaro K, Sullivan JM, Guthikonda M, et al. Acute administration of ethanol reduces apoptosis following ischemic stroke in rats. Neurosci Res. 2013; 76, 93-97. https://doi.org/10. 1016/j.neures.2013.02.011 PMID: 23511554 
42. Lages EJ, Costa FO, Cortelli SC, Cortelli JR, Cota LO, Cyrino RM, et al. Alcohol Consumption and Periodontitis: Quantification of Periodontal Pathogens and Cytokines. J Periodontol. 2015; 86, 1058-1068. https://doi.org/10.1902/jop.2015.150087 PMID: 26062839

43. Bezerra MM, de Lima V, Alencar VB, Vieira IB, Brito GA, Ribeiro RA, et al. Selective eyclooxygenase-2 inhibition prevents alveolar bone loss in experimental periodontitis in rats. J Periodontol. 2000; 71:1009-14. https://doi.org/10.1902/jop.2000.71.6.1009 PMID: 10914805

44. Kuhr A, Popa-Wagner A, Schmoll H, Schwahn C, Kocher T. Observations on experimental marginal periodontitis in rats. J Periodontal Res. 2004; 39, 101-106. https://doi.org/10.1111/j.1600-0765.2004 00710.x PMID: 15009517

45. Souza DM, Rocha R. Low caloric value of ethanol itself increases alveolar bone loss in ligature-induced periodontitis in male rats. Braz Oral Res. 2009; 23, 460-466. https://doi.org/10.1590/s180683242009000400017 PMID: 20027455

46. Souza DM, Ricardo LH, Rocha LF. Effects of alcohol intake in periodontitis progression in female rats a histometric study. Braz J Oral Sci. 2013; 13, 229-234.

47. Cochran DL. Inflammation and bone loss in periodontal disease. J Periodontol. 2008; 79, 1569-1576. https://doi.org/10.1902/jop.2008.080233 PMID: 18673012

48. Dyer SA, Buckendahl $\mathrm{P}$, Sampson HW. Alcohol consumption inhibits osteoblastic cell proliferation and activity in vivo. Alcohol. 1998; 16, 337-341. https://doi.org/10.1016/s0741-8329(98)00025-1 PMID: 9818987

49. litsuka N, Hie M, Nakanishi A, Tsukamoto I. Ethanol increases osteoclastogenesis associated with the increased expression of RANK, PU.1 and MITF in vitro and in vivo. Int J Mol Med. 2012; 30, 165-172. https://doi.org/10.3892/ijmm.2012.974 PMID: 22576626 